\title{
Review Article \\ The Role of Forkhead Box Protein M1 in Breast Cancer Progression and Resistance to Therapy
}

\author{
Raya Saba, ${ }^{1}$ Alhareth Alsayed, ${ }^{2}$ James P. Zacny, ${ }^{2}$ and Arkadiusz Z. Dudek ${ }^{2}$ \\ ${ }^{1}$ Presence Saint Joseph Hospital, 2900 North Lakeshore Drive, Chicago, IL 60657, USA \\ ${ }^{2}$ University of Illinois Hospital and Health Sciences System, UI Cancer Center, Medical Center Administration Building (M/C 700), \\ 914 South Wood Street, Chicago, IL 60612, USA
}

Correspondence should be addressed to Arkadiusz Z.Dudek; adudek@uic.edu

Received 21 September 2015; Accepted 10 January 2016

Academic Editor: Claudio Luparello

Copyright (C) 2016 Raya Saba et al. This is an open access article distributed under the Creative Commons Attribution License, which permits unrestricted use, distribution, and reproduction in any medium, provided the original work is properly cited.

\begin{abstract}
The Forkhead box M1 (FOXM1) is a transcription factor that has been implicated in normal cell growth and proliferation through control of cell cycle transition and mitotic spindle. It is implicated in carcinogenesis of various malignancies where it is activated by either amplification, increased stability, enhanced transcription, dysfunction of regulatory pathways, or activation of PI3K/AKT, epidermal growth factor receptor, Raf/MEK/MAPK, and Hedgehog pathways. This review describes the role of FOXM1 in breast cancer. This includes how FOXM1 impacts on different subtypes of breast cancer, that is, luminal/estrogen receptor positive $(\mathrm{ER}+)$, expressing human epidermal growth factor receptor 2 (HER2), basal-like breast cancer (BBC), and triple negative breast cancer (TNBC). The review also describes different tested preclinical therapeutic strategies targeting FOXM1. Developing clinically applicable therapies that specifically inhibit FOXM1 activity is a logical next step in biomarker-driven approaches against breast cancer but will not be without its challenges due to the unique properties of this transcription factor.
\end{abstract}

\section{Introduction}

The Forkhead box family is a group of over 50 mammalian proteins that is characterized by a winged helix DNA-binding domain [1]. Forkhead box M1 (FOXM1) is a transcription factor that has been well studied and implicated in normal cell growth as well as carcinogenesis. A growing body of evidence suggests a role for FOXM1 in various malignancies including breast [2], liver [3], lung [4], prostate [5], and colorectal cancer [6]. More importantly, different pathways have been elucidated but the exact mechanism remains unclear as multiple transcription factors and downstream regulatory genes are being discovered [7].

The focus of this review will be on the correlation between FOXM1 and (1) hormone and growth factor receptors pathways associated with breast cancer and (2) resistance to breast cancer therapies. We will first briefly discuss the structure of FOXM1 and its role in both normal cell functioning and carcinogenesis.

\section{FoxM1 Structure}

Fox proteins share a DNA-binding domain, the Forkhead box or winged helix domain (WHD); it consists of three helices, three sheets, and two loops or wings forming a helixturn helix-like motif. Structural variations occur between the second and third helices, whereas the helices and sheets are highly conserved. DNA binding occurs primarily through the third helix, or recognition helix, and the second wing, which bind to the major groove and minor groove of the DNA, respectively [8].

FOXM1 protein binds the core consensus sequence (A/C)AAACAAAC [9] and, in some instances, requires interaction with other factors for DNA-binding and transcriptional activity [10]. Additionally, FOXM1 may also recruit cyclin-dependent kinase (CDK) cyclin complexes through the LXL motif. Overall, there is a multitude of factors that play a role in the transcription and target gene expression of Fox proteins, including histone deacetylase (HDAC) and RUNX [11]. 
The FOXM1 gene is located on the chromosomal band $12 \mathrm{p} 13$ [12]. It consists of seven (I-VII) exons plus two alternatively expressed exons (A1 and A2) generating three alternatively spliced coding isoforms: FOXM1a, FOXM1b, and FOXM1c. Exon A2 has been shown to be a transcriptional repressor, whereas exon A1 does not have any apparent transcriptional significance. FOXM1b is lacking exons A1 and A2 sequences [9].

\section{Role of FOXM1 in Normal Cell Functioning and in Carcinogenesis}

3.1. FOXM1 in Normal Cells. Studies have shown that FoxM1 exerts its effect through regulation of the cell cycle transition points. It peaks at G1/S and G2/M phases and is essential for mitosis through promotion of $M$ phase entry $[9,13]$. FOXM1 is located in the cytoplasm at late G1 and S phases [14]. It is activated by mammalian mitotic kinase Pololike kinase-1 (Plk1) through binding to carboxyl terminal domain of FOXM1 and through further phosphorylation of two residues on this domain by cyclin-dependent kinase 1 (Cdk1) [15], cyclin E-CDK2 [16], and Raf-MEK-ERKmediated phosphorylation [14]. FoxM1 has been shown to regulate the transcription of genes involved in cell cycle by binding to promoter of cyclin D1 and cyclin B1 genes [17] and by direct activation of transcription of the Cdc25B phosphatase promoter region [18]. In the hepatoblasts of Foxmlb -/- murine embryos mitosis was associated with decreased expression of the Aurora B kinase and Polo-like kinase 1 (Plk1) and Cdc25A phosphatase [19]. Wang et al. showed, using quantitative chromatin immunoprecipitation and expression, that FoxM1 is necessary for transcription of the Aurora B kinase, survivin, centromere protein A (CENPA), and CENPB [20]. Other mechanisms of action include upregulation of transcription of $S$ phase kinaseassociated protein 2 (SKP2) and cyclin kinase subunit 1 (CKS1), subunits of the SKP1-Cullin 1-F-box ubiquitin ligase complex, leading to degradation of p27 and p21 [20]. In vitro experiments in mice detected FoxM1 in all proliferating cells, both adult and embryonic; once the cell had differentiated, it was noted that the expression of FoxM1 had significantly decreased [21]. The significance of FoxM1 in normal cell growth is supported by Krupczak-Hollis et al. [19] and Kim et al. [22], who showed that absence of FoxM1 in embryonic cells led to major developmental defects in the liver and lung and was ultimately fatal. Wonsey et al. [23] supported this notion by demonstrating that the loss of FoxM1 led to genetic mitotic spindle defects, delayed cells in mitosis, and induced mitotic catastrophe. FoxM1 transcriptional activity is controlled by the Small Ubiquitin-Like Modifier-2 (SUMO2) protein. This modification of FoxM1 peaks during G2 and M phase. SUMOylation blocks the dimerization of FoxM1, thereby alleviating FoxM1 autorepression [24].

3.2. FOXM1 in Carcinogenesis. The first evidence of a correlation between FOXM1 and human cancer was reported by Teh et al. in 2002 [25]. Their data revealed a high level of FOXM1 expression in human basal cell carcinomas, as a downstream target of the oncogenic transcription factor, glioma-associated oncogene homolog 1 (GLI1). Halasi and Gartel [26] suggested several mechanisms that would explain the increased expression and activity of FoxM1 in cancer: (1) FoxM1 locus amplification as seen in both solid [27] and hematologic malignancies [28], (2) a high level of stability or expression of FoxM1 in cancer cells initiated through different pathways, (3) enhanced transcription of FoxM1 through promoter binding of various factors such as E2 transcription factor (E2F), c-Myc, and hypoxia-inducible factor- (HIF-) 1, (4) mutations of the tumor suppressor p53 $[29,30]$, and (5) activation of FoxM1 by oncogenic signaling pathways such as PI3/Akt, epidermal growth factor receptor (EGFR), Raf/MEK/MAPK, and Hedgehog. Recently Wei et al. [31] demonstrated direct activation by FoxM1 of SNAIL gene, a key regulator of epithelial-mesenchymal transition (EMT), and showed a direct correlation with expressions of FoxM1 and Snail transcription factor in human lung adenocarcinoma tissues.

The development of metastatic disease is one of the essential hallmarks of carcinogenesis [32]. Studies have shown that FoxM1 plays a critical role in not only tumor metastasis, EMT, cell motility, invasion, and premetastatic niche formation [33] but other key cancer hallmarks as well (Figure 1) - reprogramming of energy metabolism; promotion of genomic instability [34, 35], inflammation, cell proliferation, and angiogenesis; evasion of growth/tumor suppression; circumvention of apoptosis; and enabling of replicative mortality [29].

\section{Role of FOXM1 in Breast Cancer}

4.1. FoxM1 in the Biology of Breast Cancer. The effect of FOXM1 on biology of breast cancer was evaluated in 236 women with breast cancer. Expression of FOXM1 was associated with larger tumor size, lymphovascular invasion, lymph nodes metastases, and higher stage of breast cancer. There was no significant impact of FoxM1 expression on survival when all breast cancer histologies were analyzed; however, in a subgroup of patients with estrogen receptor (ER) positive tumors, low FOXM1 expression correlated with better survival (hazard [low versus high] $=7.304,95 \%$ confidence interval [0.897-59.45], $p=0.063$ ) [36]. In another study of 501 ER positive tumors, FOXM1 was overexpressed in $20 \%$ of tumors that correlated with higher recurrence rate and shorter survival $(p=0.03)$, contributed to association of FOXM1 with stem-cell like population, increased expression of markers of EMT, and increased resistance to tamoxifen therapy [37].

The complex and diverse ways in which FOXM1 promotes breast cancer tumorigenesis are depicted in Figure 2. Yang et al. demonstrated that FOXM1 promoted EMT in breast cancer by binding and activation of the promoter of SLUG gene [38]. Xue et al. showed that FOXM1 promotes breast cancer metastases by activation of the TGF- $\beta$ pathway through interaction with SMAD3 (this prevented E3 ubiquitin-protein ligase transcriptional intermediary factor $1 \gamma$ [TIF1 $\gamma$ ] binding to SMAD3 and protected SMAD4 from ubiquitination) that leads to stabilization of the SMAD3/SMAD4 complex [39]. 


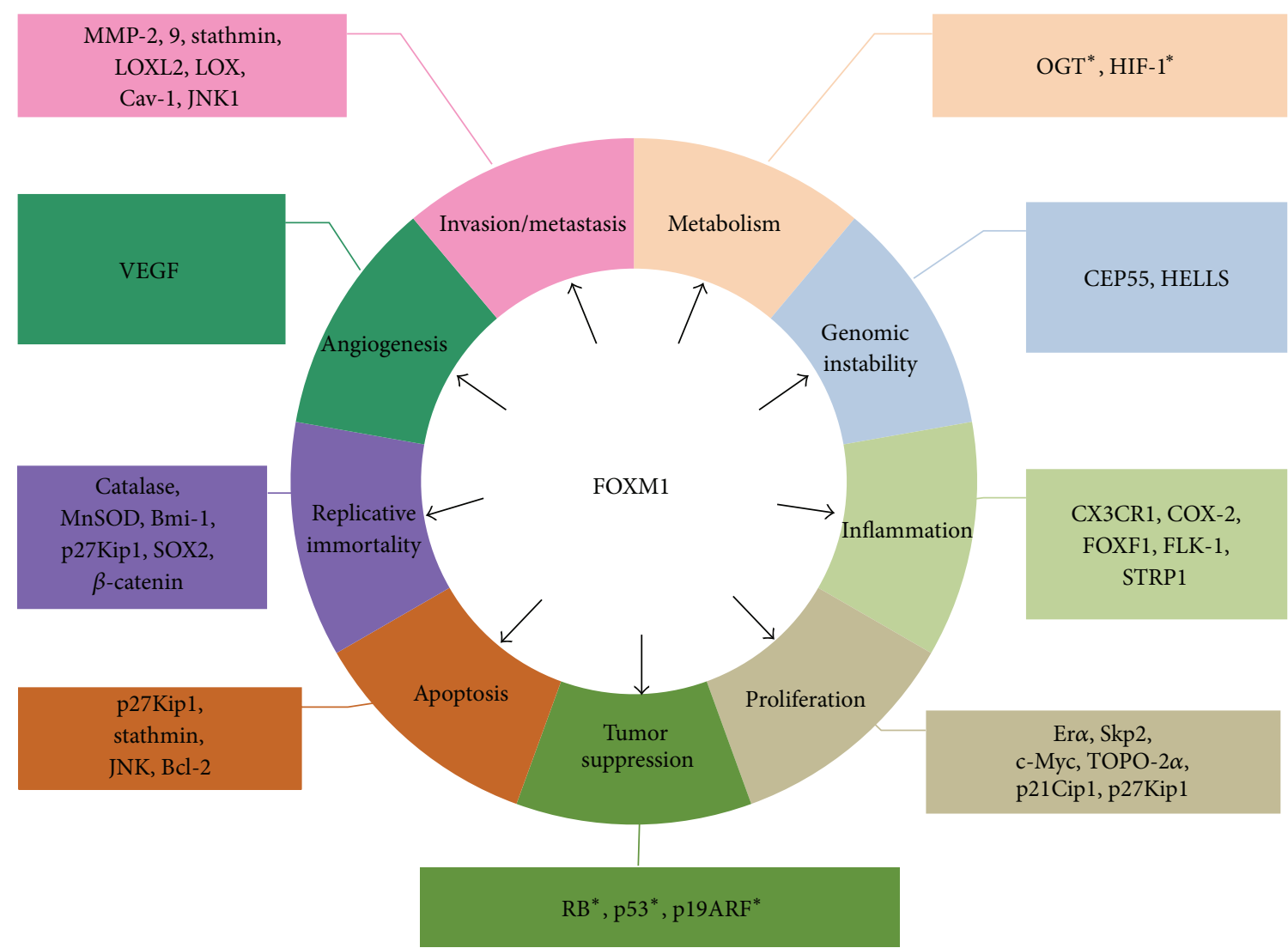

FIGURE 1: Schematic diagram of the biological role of FOXM1 in carcinogenesis. Recent research has shown that FOXM1 has a complex and diverse role in carcinogenesis. The effects of FOXM1 are mediated by numerous downstream targets as shown in this figure. In addition, several effects of FOXM1 on carcinogenesis are mediated by upstream regulators of FOXM1 ( $\left.{ }^{*}\right)$. Bcl-2: B-cell lymphoma/leukemia-2 protein; Bmi-1: B lymphoma Mo-MLV insertion region 1 homolog; Cav-1: caveolin-1; CEP55: centrosomal protein $55 \mathrm{kDa}$; c-Myc: myelocytomatosis viral oncogene homolog; COX-2: cyclooxygenase-2; CX3CR1: chemokine receptor CX3CR1; ER $\alpha$ : estrogen receptor alpha; FLK-1: fetal liver kinase-1; FOXF1: Forkhead box protein F1; FOXM1: human Forkhead box M1; HELLS: lymphoid specific helicase; HIF-1: hypoxia-inducible factor-1; JNK1: C-Jun $\mathrm{NH}_{2}$-terminal kinase-1; LOX: lysyl oxidase; LOXL2: lysyl oxidase homolog 2; MMP-2: matrix metallopeptidase-2; MMP9: matrix metallopeptidase-9; MnSOD: manganese superoxide dismutase; OGT: $O$-linked $\beta$ - $N$-acetylglucosamine transferase; p19ARF: p19 alternate reading frame; $\mathrm{p} 21^{\mathrm{Cip} 1}$ : cyclin-dependent kinase inhibitor $1 ; \mathrm{p} 27^{\mathrm{Kip} 1}$ : p27 kinase inhibitor protein 1; RB: retinoblastoma; Skp2: S phase kinase-associated protein 2; SOX2: Sry-related HMG box2; STRP1: short tandem repeat polymorphism 1; TOPO-2 $\alpha$ : topoisomerase-2 alpha; VEGF: vascular endothelial growth factor.

FOXM1 also has a role in modulation of the extracellular matrix by affecting levels of uPA, uPAR, MMP-2, MMP9, and VEGF [40]. The mechanism of VEGF induction by FOXM1 was elucidated by Karadedou et al., in which they demonstrated that FOXM1 binds to Forkhead response element in VEGF promoter [41]. Yu et al. showed that binding of FOXM1 to platelet derived growth factor-A promoter led to activation of the AKT pathway and increased breast cancer tumorigenesis [42]. Nestal de Moraes et al. [43] have shown that FOXM1 upregulates antiapoptotic genes XIAP and survivin by interacting with their promoters, contributing to chemoresistance of breast cancer cells to docetaxel, paclitaxel, and epirubicin. Moreover, coexpression of FOXM1, survivin, and nuclear XIAP was associated with poor outcomes of women with stage III breast cancer with significantly reduced 5- and 10-year survival rates versus women with tumors without these features.
Breast cancer encompasses a heterogeneous group of entities that vary greatly in terms of histology, therapy, and prognosis. This notion was suggested by Sørlie et al. [44] and later confirmed in the cancer genome atlas published in 2012 [45]. Human breast malignancies can be divided into five subtypes: normal breast-like, luminal A, luminal B, HER2/Neu-enriched, and basal-like breast cancer (BBC).

4.2. FOXM1 and Luminal/Estrogen Receptor Positive (ER+) Breast Cancer. The effect of estrogen on both normal and malignant breast tissue is mediated through two types of estrogen receptors (ER): ER $\alpha$ and $E R \beta$. Although they bind to estrogen with equal affinity, $\mathrm{ER} \alpha$ and $\mathrm{ER} \beta$ respond differently to estrogen stimulation: activated $\operatorname{ER} \alpha$ induces breast epithelium proliferation, whereas $\operatorname{ER} \beta$ has antiproliferative and proapoptotic effects [46]. Approximately $70 \%$ of breast cancers are estrogen receptor positive $(\mathrm{ER}+)$ and these 


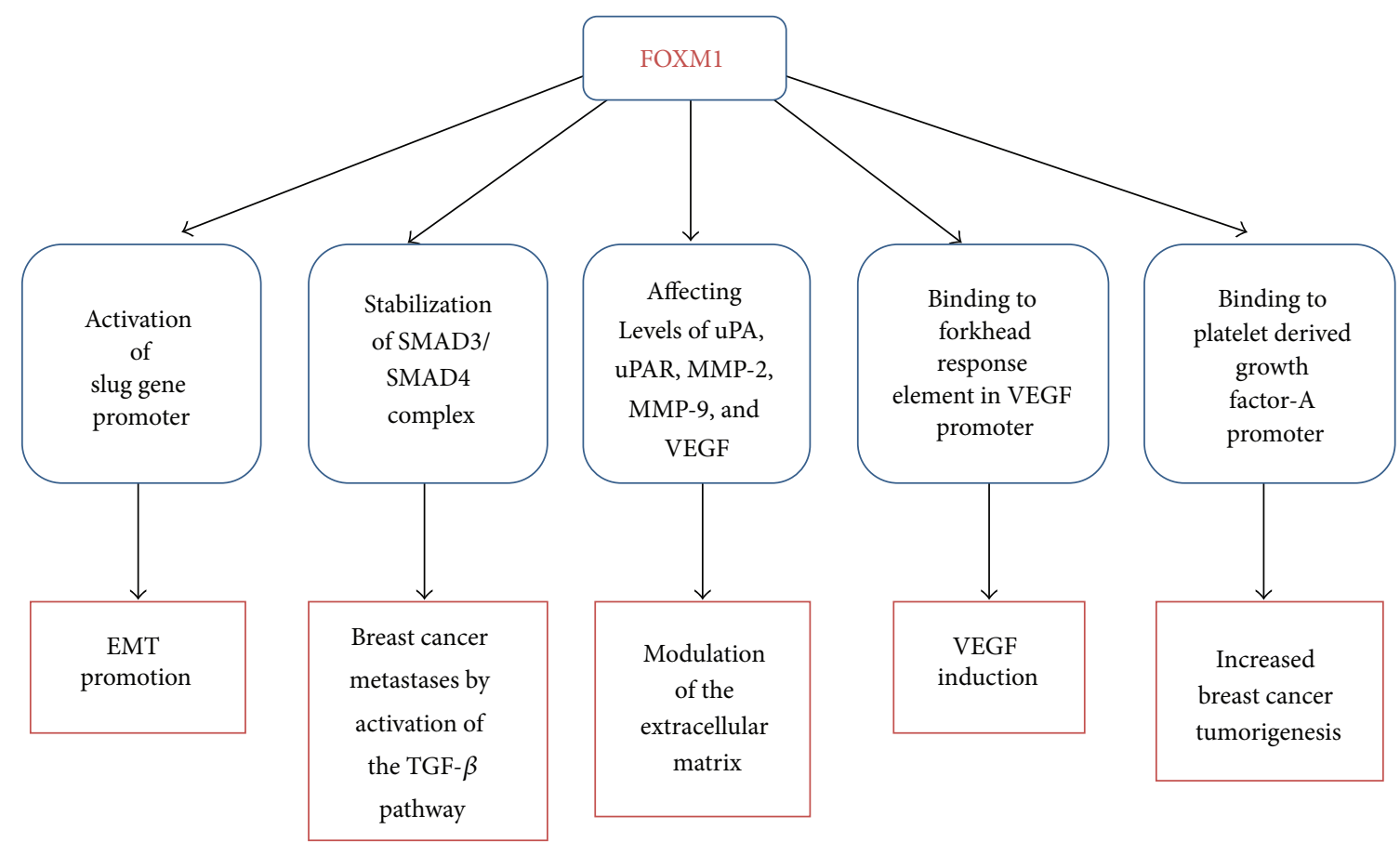

FIGURE 2: Schematic diagram of the biological role of FOXM1 in breast cancer tumorigenesis. Recent research has shown that the effects of FOXM1 on breast cancer tumorigenesis are mediated by a number of diverse biological mechanisms. EMT: epithelial-mesenchymal transition; TGF- $\beta$ : transforming growth factor-beta; uPA: urokinase; uPAR: urokinase receptor; MMP-2: matrix metallopeptidase-2; MMP-9: matrix metallopeptidase-9; VEGF: vascular endothelial growth factor.

tumors are often better differentiated, grow slowly, and have a favorable prognosis [47].

Early research about the Forkhead box family and growth factors in breast cancer emerged in 2000. Jackson et al. provided the first evidence of a link between epidermal growth factor (EGF) and a Forkhead box family member, FOXO3a, known at that time as FKHR [48]. Influenced by these findings, Madureira et al. [49] and Karadedou [50] found a positive correlation between ER $\alpha$ and FOXM1 and an inverse correlation between ER $\alpha$ and FOXO3a. Their results showed that FOXM1 is a physiologic regulator of $\mathrm{ER} \alpha$ expression in breast cancer cells, both at the protein and at mRNA levels. Consistent with these findings, later studies confirmed the tumor suppressive qualities of FOXO3a [51], including cases of ER+ breast malignancies [52]. Carr et al. reported transcriptional repressor function of FOXM1 inhibiting differentiation of luminal progenitor cells by inducing methylation of promoter of the zinc finger transcription factor GATA-3 through association with DNMT3b [53]. Millour et al. supported the critical role of FOXM1 in mitogenic functions of ER $\alpha$ and estrogen in breast malignancies [54]. Additionally, they showed that FOXM1 deregulation may also contribute to antiestrogen insensitivity, opening the door to practical applications of these findings in the field of therapeutics. Whereas the focus of these studies was on $\mathrm{ER} \alpha$, Horimoto et al. studied the effects of ER $\beta$ expression on breast cancer [55]. Their investigations showed that ER $\beta$ had an antiproliferative effect through repression of FOXM1 expression in breast cancer cells; this effect was mediated by an estrogen-response element within the proximal promoter region that is also a target of ER $\alpha$.

The correlation between FOXM1 and ER+ breast cancer has also been shown on the genetic level. Ahn et al. created a 70-gene signature which was found to be of prognostic value in ER+ breast cancer patients in that FOXM1 suggested poorer prognosis [56]. This signature was found to be most helpful in cases of intermediate Oncotype recurrence scores. Other studies included genome-wide mapping by Sanders et al. which revealed a direct relationship between FOXM1 and $\mathrm{ER} \alpha$ [57]. Their analysis uncovered another signature of 38 FOXM1-regulated genes with a prognostic value.

4.3. FOXM1 and Human Epidermal Growth Factor Receptor 2 (HER2). The human epidermal growth factor receptor 2 (HER2) is a transmembrane tyrosine kinase receptor and a member of the epidermal growth factor (EGF) family [58]. The amplification of HER2 can be seen in up to $25 \%$ of breast cancer cases and has been shown to correlate with high relapse rates and poor survival [59]. In 2008, Bektas et al. provided the first evidence of a positive correlation between HER2 status and FOXM1 expression in breast cancer specimens, in comparison to normal breast tissue [60]. Additionally, their work verified the overexpression of FOXM1 in breast cancer on both the RNA and protein level. Francis et al. elaborated on the concept further through various in vitro and in vivo experiments [61]. Their work suggested that FOXM1 may in fact be a downstream target and marker of HER2 overexpression in breast cancer. This study also had 
translational implications by showing a suppressive role for lapatinib (a HER2 inhibitor) on FOXM1 expression at the protein, mRNA, and gene promoter levels. Subsequent data showed a role for FOXM1 in the development of trastuzumab (a HER2 antibody) resistance [62]. This is particularly important because trastuzumab resistance may develop quickly and represents a challenge in the treatment of breast cancer.

\subsection{FOXM1, Basal-Like Breast Cancer (BBC), and Triple Neg-} ative Breast Cancer (TNBC). Basal-like breast cancer (BBC) is an aggressive phenotype of breast malignancies that is often associated with poor prognosis [63]. BBC cells lack hormone estrogen receptors (ER) and progesterone receptors (PR) and express genes that are usually seen in basal or myoepithelial cells of normal breast tissue. Triple negative breast cancers (TNBC), on the other hand, are tumors that lack HER2 in addition to ER and PR. Although differences between these two types of breast cancer have been demonstrated, BBC and TNBC overlap significantly [64]. These tumors represent a therapeutic challenge because of lack of effective therapy. Although TNBC accounts for only $15 \%$ of breast cancer subtypes, it causes $25 \%$ of breast cancer-related deaths due to its aggressive and refractory nature [65].

EMT has been correlated with TNBC [66], and NFkappaB is a transcription factor that has been shown to be essential in EMT in breast cancer [67]. Arora et al. incorporated these two concepts in their search for a therapeutic agent for TNBC [68]. They discovered that panepoxydone (PP), a NF-kappaB inhibitor, halted proliferation, induced apoptosis, and reversed EMT in breast cancer, in particular TNBC. Interestingly, their work revealed a downregulatory effect of PP on FOXM1 as well. A whole genome and transcriptome sequencing of TNBC cells by Craig et al. revealed consistent overexpression of FOXM1 in TNBC [69]. The role of FOXM1 has also been seen in BBC cancers, including FOXM1-dependent overexpression of MELK, a novel oncogenic kinase [70].

\section{FOXM1 and Breast Cancer Therapeutics}

Taking into consideration the significant role of FOXM1 in breast cancer biology this transcription factor could become an attractive target for cancer treatment. Herein, we describe some breast cancer therapeutic strategies targeting FOXM1.

A FOXM1-specific small interfering RNA (siRNA) was found to be effective in reducing the expression of FOXM1 proteins in vivo [71]. Treatment of breast cancer cells with adenoviral vector expressing short hairpin downregulating FOXM1 led to inhibition of breast cancer tumor formation [72]. Overexpression of microRNA miR802 led to downregulation of FOXM1 and inhibited proliferation of breast cancer cells [73]. A thiazole ring containing thiostrepton, an antibiotic with antitumor activities [74], was shown to induce arrest and death of breast cancer cells through downregulating FOXM1 expression [75]. As stated earlier [24], SUMO is a posttranslational modifier that is essential for activation of FOXM1. A SUMOylation protease, sentrin-specific protease 2 (SENP2), significantly decreased SUMOylation of FOXM1 and interfered with its function [76]. Casticin, an active ingredient derived from Fructus Viticis, the fruit of a traditional Chinese medicine, has anticarcinogenic activity in breast cancer [77]. Recent experiments showed that it can induce apoptosis of breast cancer cells by reducing the expression of FOXM1 [78].

FOXM1 has been shown to play a critical role in development of resistance to breast cancer therapeutics. FOXM1 is a downstream target of 14-3-3 , a marker of endocrine therapy resistance in breast cancer malignancy [79]. FOXM1 was found to contribute to cisplatin (a platinum agent) resistance in breast cancer cells. The effect was thought to be mediated by the enhancement of DNA-damage repair pathways and the promotion of cell cycle progression or inhibition of cell cycle checkpoints and apoptosis [80]. FOXM1 overexpression was implicated in the resistance to trastuzumab (a HER2 monoclonal antibody) and paclitaxel (a microtubule stabilizing agent). Treatment with a siRNA targeting FOXM1 or an alternate reading frame- (ARF-) derived peptide resulted in improved therapeutic sensitivity to these agents [81]. FOXM1 caused doxorubicin resistance in breast cancer by enhancing DNA repair. The nuclear factor NF-kappa-B1 (NF $\kappa \mathrm{B} 1)$ interacted with FOXM1 in the presence of doxorubicin to protect breast cancer cells from DNA damage [82]. Epirubicin could activate ataxia-telangiectasia mutated (ATM) that promotes E2F activity and FOXM1 expression [83]. In addition, a study by de Olano et al. showed that the mechanism of epirubicin resistance was mediated by activation of mitogen-activated protein kinase-activated protein kinase 2 leading to increased phosphorylation of transcription factor E2F1 at Ser-364 resulting in increased FOXM1 expression [84]. Khongkow et al. demonstrated that FOXM1 reduced senescence induced by epirubicin, by increasing expression of NBS1 leading to enhanced homologous recombination DNA repair [85]. Another work by Khongkow et al. postulated that resistance to paclitaxel, a tubulin targeting agent, can be mediated by FOXM1 through enhancement of promoter activity of transcriptional activity of KIF20A [86]. Both FOXM1 and KIF20A are critical for normal formation of mitotic spindle and thus could interfere with paclitaxel activity. Nestal de Moraes et al. [43] demonstrated that resistance to epirubicin, docetaxel, and paclitaxel was associated with activation of XIAP and survivin by direct interaction of FOXM1 with promoters of these antiapoptotic genes.

\section{Conclusion}

Forkhead box M1 plays a significant role in breast carcinogenesis, disease progression to metastatic stage, and development of resistance to subsequent cancer therapy, and thus it could be an attractive target for therapeutic interventions in this malignancy. However, due to incomplete understanding of the biology of the FOX family of transcription factors that has complex regulatory mechanisms, it has been a challenge to develop a drug that functions specifically as a FOXM1 inhibitor [87]. In addition, druggability of FOXM1 has been taxing because of lack of substrate binding pockets and hydrophobic surfaces [88]. A recent discovery through high throughput fluorescence polarization assay of a novel small 
molecule that specifically inhibits FOXM1 interaction with DNA may be a first breakthrough in the design of selective inhibitors of this transcription factor [89]. Once FOXM1 targeted agents are available, appropriate clinical testing in different breast cancer subtypes is warranted, most likely in a biomarker-driven setting. At present, however, there is no clear biomarker developed to assess sensitivity of breast cancer to FOXM1 inhibition. This could be a goal of early pilot studies, especially in the most challenging breast cancer subtype, triple negative disease.

\section{Conflict of Interests}

The authors have no conflict of interests to report.

\section{Acknowledgment}

The authors thank Pradip Raychaudhuri, Ph.D., for his valuable advice regarding the paper.

\section{References}

[1] K. H. Kaestner, W. Knöchel, and D. E. Martínez, "Unified nomenclature for the winged helix/forkhead transcription factors," Genes \& Development, vol. 14, no. 2, pp. 142-146, 2000.

[2] C. M. Perou, T. Sørile, M. B. Eisen et al., "Molecular portraits of human breast tumours," Nature, vol. 406, no. 6797, pp. 747-752, 2000.

[3] V. V. Kalinichenko, M. L. Major, X. Wang et al., "Foxmlb transcription factor is essential for development of hepatocellular carcinomas and is negatively regulated by the p19ARF tumor suppressor," Genes \& Development, vol. 18, no. 7, pp. 830-850, 2004.

[4] I.-M. Kim, T. Ackerson, S. Ramakrishna et al., "The Forkhead Box $\mathrm{ml}$ transcription factor stimulates the proliferation of tumor cells during development of lung cancer," Cancer Research, vol. 66, no. 4, pp. 2153-2161, 2006.

[5] T. V. Kalin, I.-C. Wang, T. J. Ackerson et al., "Increased levels of the FoxM1 transcription factor accelerate development and progression of prostate carcinomas in both TRAMP and LADY transgenic mice," Cancer Research, vol. 66, no. 3, pp. 1712-1720, 2006.

[6] Y. Yoshida, I.-C. Wang, H. M. Yoder, N. O. Davidson, and R. H. Costa, "The forkhead box M1 transcription factor contributes to the development and growth of mouse colorectal cancer," Gastroenterology, vol. 132, no. 4, pp. 1420-1431, 2007.

[7] S. S. Myatt and E. W.-F. Lam, "The emerging roles of forkhead box (Fox) proteins in cancer," Nature Reviews Cancer, vol. 7, no. 11, pp. 847-859, 2007.

[8] K. L. Clark, E. D. Halay, E. Lai, and S. K. Burley, "Co-crystal structure of the HNF-3/fork head DNA-recognition motif resembles histone H5," Nature, vol. 364, no. 6436, pp. 412-420, 1993.

[9] H. Ye, T. F. Kelly, U. Samadani et al., "Hepatocyte nuclear factor $3 /$ fork head homolog 11 is expressed in proliferating epithelial and mesenchymal cells of embryonic and adult tissues," Molecular and Cellular Biology, vol. 17, no. 3, pp. 1626-1641, 1997.

[10] S. Li, J. Weidenfeld, and E. E. Morrisey, "Transcriptional and DNA binding activity of the Foxp1/2/4 family is modulated by heterotypic and homotypic protein interactions," Molecular and Cellular Biology, vol. 24, no. 2, pp. 809-822, 2004.
[11] M. Ono, H. Yaguchi, N. Ohkura et al., "Foxp3 controls regulatory T-cell function by interacting with AML1/Runxl," Nature, vol. 446, no. 7136, pp. 685-689, 2007.

[12] W. Korver, J. Roose, K. Heinen et al., “The human TRIDENT/ HFH-11/FKHL16 gene: structure, localization, and promoter characterization," Genomics, vol. 46, no. 3, pp. 435-442, 1997.

[13] W. Korver, M. W. Schilham, P. Moerer et al., "Uncoupling of S phase and mitosis in cardiomyocytes and hepatocytes lacking the winged-helix transcription factor Trident," Current Biology, vol. 8, no. 24, pp. 1327-1330, 1998.

[14] R. Y. Ma, T. H. Tong, A. M. Cheung, A. C. Tsang, W. Y. Leung, and K. M. Yao, "Raf/MEK/MAPK signaling stimulates the nuclear translocation and transactivating activity of FOXM1c," Journal of Cell Science, vol. 118, part 4, pp. 795-806, 2005.

[15] Z. Fu, L. Malureanu, J. Huang et al., "Plk1-dependent phosphorylation of FoxM1 regulates a transcriptional programme required for mitotic progression," Nature Cell Biology, vol. 10, no. 9, pp. 1076-1082, 2008.

[16] M. L. Major, R. Lepe, and R. H. Costa, "Forkhead box M1B transcriptional activity requires binding of Cdk-cyclin complexes for phosphorylation-dependent recruitment of p300/CBP coactivators," Molecular and Cellular Biology, vol. 24, no. 7, pp. 26492661, 2004.

[17] X. Wang, E. Quail, N.-J. Hung, Y. Tan, H. Ye, and R. H. Costa, "Increased levels of forkhead box M1B transcription factor in transgenic mouse hepatocytes prevent age-related proliferation defects in regenerating liver," Proceedings of the National Academy of Sciences of the United States of America, vol. 98, no. 20, pp. 11468-11473, 2001.

[18] X. Wang, H. Kiyokawa, M. B. Dennewitz, and R. H. Costa, "The Forkhead Box mlb transcription factor is essential for hepatocyte DNA replication and mitosis during mouse liver regeneration," Proceedings of the National Academy of Sciences of the United States of America, vol. 99, no. 26, pp. 16881-16886, 2002.

[19] K. Krupczak-Hollis, X. Wang, V. V. Kalinichenko et al., "The mouse Forkhead Box $\mathrm{ml}$ transcription factor is essential for hepatoblast mitosis and development of intrahepatic bile ducts and vessels during liver morphogenesis," Developmental Biology, vol. 276, no. 1, pp. 74-88, 2004.

[20] I.-C. Wang, Y.-J. Chen, D. Hughes et al., "Forkhead box M1 regulates the transcriptional network of genes essential for mitotic progression and genes encoding the SCF (Skp2-Cks1) ubiquitin ligase," Molecular and Cellular Biology, vol. 25, no. 24, pp. 10875-10894, 2005.

[21] W. Korver, J. Roose, and H. Clevers, "The winged-helix transcription factor Trident is expressed in cycling cells," Nucleic Acids Research, vol. 25, no. 9, pp. 1715-1719, 1997.

[22] I.-M. Kim, S. Ramakrishna, G. A. Gusarova, H. M. Yoder, R. H. Costa, and V. V. Kalinichenko, "The Forkhead Box m1 transcription factor is essential for embryonic development of pulmonary vasculature," The Journal of Biological Chemistry, vol. 280, no. 23, pp. 22278-22286, 2005.

[23] D. R. Wonsey and M. T. Follettie, "Loss of the forkhead transcription factor FoxM1 causes centrosome amplification and mitotic catastrophe," Cancer Research, vol. 65, no. 12, pp. 51815189, 2005.

[24] J. Schimmel, K. Eifler, J. O. Sigurðsson et al., "Uncovering SUMOylation dynamics during cell-cycle progression reveals FoxM1 as a key mitotic SUMO target protein," Molecular Cell, vol. 53, no. 6, pp. 1053-1066, 2014. 
[25] M.-T. Teh, S.-T. Wong, G. W. Neill, L. R. Ghali, M. P. Philpott, and A. G. Quinn, "FOXM1 is a downstream target of Glil in basal cell carcinomas," Cancer Research, vol. 62, no. 16, pp. 47734780, 2002.

[26] M. Halasi and A. L. Gartel, "FOX(M1) news-it is cancer," Molecular Cancer Therapeutics, vol. 12, no. 3, pp. 245-254, 2013.

[27] J. Laoukili, J. M. Stahl, and R. H. Medema, "FoxM1: at the crossroads of ageing and cancer," Biochimica et Biophysica Acta, vol. 1775, no. 1, pp. 92-102, 2007.

[28] M. R. Green, C. Aya-Bonilla, M. K. Gandhi et al., "Integrative genomic profiling reveals conserved genetic mechanisms for tumorigenesis in common entities of non-Hodgkin's lymphoma," Genes Chromosomes and Cancer, vol. 50, no. 5, pp. 313326, 2011.

[29] B. Pandit, M. Halasi, and A. L. Gartel, "p53 negatively regulates expression of FoxM1," Cell Cycle, vol. 8, no. 20, pp. 3425-3427, 2009.

[30] A. M. Barsotti and C. Prives, "Pro-proliferative FoxM1 is a target of p53-mediated repression," Oncogene, vol. 28, no. 48, pp. 4295-4305, 2009.

[31] P. Wei, N. Zhang, Y. Wang et al., "FOXM1 promotes lung adenocarcinoma invasion and metastasis by upregulating SNAIL," International Journal of Biological Sciences, vol. 11, no. 2, pp. 186198, 2015.

[32] D. Hanahan and R. A. Weinberg, "Hallmarks of cancer: the next generation," Cell, vol. 144, no. 5, pp. 646-674, 2011.

[33] H. J. Park, G. Gusarova, Z. Wang et al., "Deregulation of FoxM1b leads to tumour metastasis," EMBO Molecular Medicine, vol. 3, no. 1, pp. 21-34, 2011.

[34] E. Gemenetzidis, A. Bose, A. M. Riaz et al., "FOXM1 upregulation is an early event in human squamous cell carcinoma and it is enhanced by nicotine during malignant transformation," PLoS ONE, vol. 4, no. 3, Article ID e4849, 2009.

[35] M.-T. Teh, E. Gemenetzidis, T. Chaplin, B. D. Young, and M. P. Philpott, "Upregulation of FOXM1 induces genomic instability in human epidermal keratinocytes," Molecular Cancer, vol. 9, article 45, 2010.

[36] H. Ahn, J. Sim, R. Abdul et al., "Increased expression of forkhead box M1 is associated with aggressive phenotype and poor prognosis in estrogen receptor-positive breast cancer," Journal of Korean Medical Science, vol. 30, no. 4, pp. 390-397, 2015.

[37] A. Bergamaschi, Z. Madak-Erdogan, Y. J. Kim, Y. L. Choi, H. $\mathrm{Lu}$, and B. S. Katzenellenbogen, "The forkhead transcription factor FOXM1 promotes endocrine resistance and invasiveness in estrogen receptor-positive breast cancer by expansion of stem-like cancer cells," Breast Cancer Research, vol. 16, no. 5, p. 436, 2014.

[38] C. Yang, H. Chen, G. Tan et al., "FOXM1 promotes the epithelial to mesenchymal transition by stimulating the transcription of Slug in human breast cancer," Cancer Letters, vol. 340, no. 1, pp. 104-112, 2013.

[39] J. Xue, X. Lin, W.-T. Chiu et al., "Sustained activation of SMAD3/SMAD4 by FOXM1 promotes TGF- $\beta$-dependent cancer metastasis," The Journal of Clinical Investigation, vol. 124, no. 2, pp. 564-579, 2014.

[40] A. Ahmad, Z. Wang, D. Kong et al., "FoxM1 down-regulation leads to inhibition of proliferation, migration and invasion of breast cancer cells through the modulation of extra-cellular matrix degrading factors," Breast Cancer Research and Treatment, vol. 122, no. 2, pp. 337-346, 2010.
[41] C. T. Karadedou, A. R. Gomes, J. Chen et al., "FOXO3a represses VEGF expression through FOXM1-dependent and independent mechanisms in breast cancer," Oncogene, vol. 31, no. 14, pp. 1845-1858, 2012.

[42] G. Yu, A. Zhou, J. Xue et al., "FoxM1 promotes breast tumorigenesis by activating PDGF-A and forming a positive feedback loop with the PDGF/AKT signaling pathway," Oncotarget, vol. 6, no. 13, pp. 11281-11294, 2015.

[43] G. Nestal de Moraes, D. Delbue, K. L. Silva et al., "FOXM1 targets XIAP and Survivin to modulate breast cancer survival and chemoresistance," Cellular Signalling, vol. 27, no. 12, pp. 2496-2505, 2015.

[44] T. Sørlie, C. M. Perou, R. Tibshirani et al., "Gene expression patterns of breast carcinomas distinguish tumor subclasses with clinical implications," Proceedings of the National Academy of Sciences of the United States of America, vol. 98, no. 19, pp. 1086910874, 2001.

[45] Cancer Genome Atlas Network, "Comprehensive molecular portraits of human breast tumours," Nature, vol. 490, no. 7418, pp. 61-70, 2012.

[46] V. Speirs and R. A. Walker, "New perspectives into the biological and clinical relevance of oestrogen receptors in the human breast," The Journal of Pathology, vol. 211, no. 5, pp. 499-506, 2007.

[47] P. L. Fitzgibbons, D. L. Page, D. Weaver et al., "Prognostic factors in breast cancer. College of American Pathologists Consensus Statement 1999," Archives of Pathology \& Laboratory Medicine, vol. 124, no. 7, pp. 966-978, 2000.

[48] J. G. Jackson, J. I. Kreisberg, A. P. Koterba, D. Yee, and M. G. Brattain, "Phosphorylation and nuclear exclusion of the forkhead transcription factor FKHR after epidermal growth factor treatment in human breast cancer cells," Oncogene, vol. 19, no. 40, pp. 4574-4581, 2000.

[49] P. A. Madureira, R. Varshochi, D. Constantinidou et al., "The forkhead box M1 protein regulates the transcription of the estrogen receptor $\alpha$ in breast cancer cells," The Journal of Biological Chemistry, vol. 281, no. 35, pp. 25167-25176, 2006.

[50] C. T. Karadedou, "Regulation of the FOXM1 transcription factor by the estrogen receptor alpha at the protein level, in breast cancer," Hippokratia, vol. 10, no. 3, pp. 128-132, 2006.

[51] J.-H. Paik, R. Kollipara, G. Chu et al., "FoxOs are lineagerestricted redundant tumor suppressors and regulate endothelial cell homeostasis," Cell, vol. 128, no. 2, pp. 309-323, 2007.

[52] Y. Zou, W.-B. Tsai, C.-J. Cheng et al., "Forkhead box transcription factor FOXO3a suppresses estrogen-dependent breast cancer cell proliferation and tumorigenesis," Breast Cancer Research, vol. 10, no. 1, article R21, 2008.

[53] J. R. Carr, M. M. Kiefer, H. J. Park et al., "FoxM1 regulates mammary luminal cell fate," Cell Reports, vol. 1, no. 6, pp. 715$729,2012$.

[54] J. Millour, D. Constantinidou, A. V. Stavropoulou et al., "FOXM1 is a transcriptional target of ER $\alpha$ and has a critical role in breast cancer endocrine sensitivity and resistance," Oncogene, vol. 29, no. 20, pp. 2983-2995, 2010.

[55] Y. Horimoto, J. Hartman, J. Millour et al., "ER $\beta 1$ represses FOXM1 expression through targeting ER $\alpha$ to control cell proliferation in breast cancer," The American Journal of Pathology, vol. 179, no. 3, pp. 1148-1156, 2011.

[56] S. G. Ahn, H. M. Lee, H. W. Lee et al., "Prognostic discrimination using a 70-gene signature among patients with estrogen receptor-positive breast cancer and an intermediate 21-gene 
recurrence score," International Journal of Molecular Sciences, vol. 14, no. 12, pp. 23685-23699, 2013.

[57] D. A. Sanders, C. S. Ross-Innes, D. Beraldi, J. S. Carroll, and S. Balasubramanian, "Genome-wide mapping of FOXM1 binding reveals co-binding with estrogen receptor alpha in breast cancer cells," Genome Biology, vol. 14, no. 1, article R6, 2013.

[58] D. F. Stern, P. A. Heffernan, and R. A. Weinberg, "P185, a product of the neu proto-oncogene, is a receptorlike protein associated with tyrosine kinase activity," Molecular and Cellular Biology, vol. 6, no. 5, pp. 1729-1740, 1986.

[59] D. J. Slamon, G. M. Clark, S. G. Wong, W. J. Levin, A. Ullrich, and W. L. McGuire, "Human breast cancer: correlation of relapse and survival with amplification of the HER-2/neu oncogene," Science, vol. 235, no. 4785, pp. 177-182, 1987.

[60] N. Bektas, A. ten Haaf, J. Veeck et al., "Tight correlation between expression of the Forkhead transcription factor FOXM1 and HER2 in human breast cancer," BMC Cancer, vol. 8, article 42, 2008.

[61] R. E. Francis, S. S. Myatt, J. Krol et al., "FoxM1 is a downstream target and marker of HER2 overexpression in breast cancer," International Journal of Oncology, vol. 35, no. 1, pp. 57-68, 2009.

[62] J. R. Carr, H. J. Park, Z. Wang, M. M. Kiefer, and P. Raychaudhuri, "FoxM1 mediates resistance to herceptin and paclitaxel," Cancer Research, vol. 70, no. 12, pp. 5054-5063, 2010.

[63] E. A. Rakha, J. S. Reis-Filho, and I. O. Ellis, "Basal-like breast cancer: a critical review," Journal of Clinical Oncology, vol. 26, no. 15 , pp. 2568-2581, 2008.

[64] W. D. Foulkes, I. E. Smith, and J. S. Reis-Filho, "Triple-negative breast cancer," The New England Journal of Medicine, vol. 363, no. 20, pp. 1938-1948, 2010.

[65] F. Rastelli, S. Biancanelli, A. Falzetta et al., "Triple-negative breast cancer: current state of the art," Tumori, vol. 96, no. 6, pp. 875-888, 2010.

[66] H. Jeong, Y.-J. Ryu, J. An, Y. Lee, and A. Kim, "Epithelialmesenchymal transition in breast cancer correlates with high histological grade and triple-negative phenotype," Histopathology, vol. 60, no. 6, pp. E87-E95, 2012.

[67] M. A. Huber, N. Azoitei, B. Baumann et al., "NF- $\kappa$ B is essential for epithelial-mesenchymal transition and metastasis in a model of breast cancer progression," The Journal of Clinical Investigation, vol. 114, no. 4, pp. 569-581, 2004.

[68] R. Arora, C. Yates, B. D. Gary et al., "Panepoxydone targets NF$\kappa \mathrm{B}$ and FOXM1 to inhibit proliferation, induce apoptosis and reverse epithelial to mesenchymal transition in breast cancer," PLoS ONE, vol. 9, no. 6, Article ID e98370, 2014.

[69] D. W. Craig, J. A. O’Shaughnessy, J. A. Kiefer et al., “Genome and transcriptome sequencing in prospective metastatic triplenegative breast cancer uncovers therapeutic vulnerabilities," Molecular Cancer Therapeutics, vol. 12, no. 1, pp. 104-116, 2013.

[70] Y. Wang, Y.-M. Lee, L. Baitsch et al., "MELK is an oncogenic kinase essential for mitotic progression in basal-like breast cancer cells," eLife, vol. 3, Article ID e01763, 2014.

[71] M. Wang and A. L. Gartel, "The suppression of FOXM1 and its targets in breast cancer xenograft tumors by siRNA," Oncotarget, vol. 2, no. 12, pp. 1218-1226, 2011.

[72] C. Yang, H. Chen, L. Yu et al., "Inhibition of FOXM1 transcription factor suppresses cell proliferation and tumor growth of breast cancer," Cancer Gene Therapy, vol. 20, no. 2, pp. 117-124, 2013.

[73] F. Yuan and W. Wang, "MicroRNA-802 suppresses breast cancer proliferation through downregulation of FoxM1," Molecular Medicine Reports, vol. 12, no. 3, pp. 4647-4651, 2015.
[74] K. C. Nicolaou, M. Zak, S. Rahimipour et al., "Discovery of a biologically active thiostrepton fragment," Journal of the American Chemical Society, vol. 127, no. 43, pp. 15042-15044, 2005.

[75] J. M.-M. Kwok, S. S. Myatt, C. M. Marson, R. C. Coombes, D. Constantinidou, and E. W.-F. Lam, "Thiostrepton selectively targets breast cancer cells through inhibition of forkhead box M1 expression," Molecular Cancer Therapeutics, vol. 7, no. 7, pp. 2022-2032, 2008.

[76] C.-M. Wang, R. Liu, L. Wang, L. Nascimento, V. C. Brennan, and W.-H. Yang, "SUMOylation of FOXM1B alters its transcriptional activity on regulation of MiR-200 family and JNK1 in MCF7 human breast cancer cells," International Journal of Molecular Sciences, vol. 15, no. 6, pp. 10233-10251, 2014.

[77] K. Haïdara, L. Zamir, Q.-W. Shi, and G. Batist, "The flavonoid Casticin has multiple mechanisms of tumor cytotoxicity action," Cancer Letters, vol. 242, no. 2, pp. 180-190, 2006.

[78] L.-P. Liu, X.-C. Cao, F. Liu, M.-F. Quan, X.-F. Sheng, and K.-Q. Ren, "Casticin induces breast cancer cell apoptosis by inhibiting the expression of forkhead box protein M1," Oncology Letters, vol. 7, no. 5, pp. 1711-1717, 2014.

[79] A. Bergamaschi, B. L. Christensen, and B. S. Katzenellenbogen, "Reversal of endocrine resistance in breast cancer: interrelationships among 14-3-3 $\zeta$, FOXM1, and a gene signature associated with mitosis," Breast Cancer Research, vol. 13, no. 3, article R70, 2011.

[80] J. M.-M. Kwok, B. Peck, L. J. Monteiro et al., "FOXM1 confers acquired cisplatin resistance in breast cancer cells," Molecular Cancer Research, vol. 8, no. 1, pp. 24-34, 2010.

[81] J. R. Carr, H. J. Park, Z. Wang, M. M. Kiefer, and P. Raychaudhuri, "FoxM1 mediates resistance to herceptin and paclitaxel," Cancer Research, vol. 70, no. 12, pp. 5054-5063, 2010.

[82] Y.-Y. Park, S. Y. Jung, N. B. Jennings et al., "FOXM1 mediates Dox resistance in breast cancer by enhancing DNA repair," Carcinogenesis, vol. 33, no. 10, pp. 1843-1853, 2012.

[83] J. Millour, N. de Olano, Y. Horimoto et al., "ATM and p53 regulate FOXM1 expression via E2F in breast cancer epirubicin treatment and resistance," Molecular Cancer Therapeutics, vol. 10, no. 6, pp. 1046-1058, 2011.

[84] N. de Olano, C.-Y. Koo, L. J. Monteiro et al., "The p38 MAPK-MK2 axis regulates E2F1 and FOXM1 expression after epirubicin treatment," Molecular Cancer Research, vol. 10, no. 9, pp. 1189-1202, 2012.

[85] P. Khongkow, U. Karunarathna, M. Khongkow et al., "FOXM1 targets NBS1 to regulate DNA damage-induced senescence and epirubicin resistance," Oncogene, vol. 33, no. 32, pp. 4144-4155, 2014.

[86] P. Khongkow, A. R. Gomes, C. Gong et al., "Paclitaxel targets FOXM1 to regulate KIF20A in mitotic catastrophe and breast cancer paclitaxel resistance," Oncogene, 2015.

[87] A. L. Gartel, "A new target for proteasome inhibitors: FoxM1," Expert Opinion on Investigational Drugs, vol. 19, no. 2, pp. 235242, 2010.

[88] G. L. Verdine and L. D. Walensky, "The challenge of drugging undruggable targets in cancer: lessons learned from targeting BCL-2 family members," Clinical Cancer Research, vol. 13, no. 24, pp. 7264-7270, 2007.

[89] M. V. Gormally, T. S. Dexheimer, G. Marsico et al., "Suppression of the FOXM1 transcriptional programme via novel small molecule inhibition," Nature Communications, vol. 5, article 5165, 2014. 


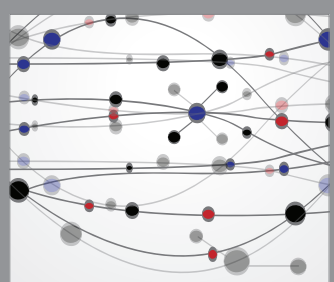

The Scientific World Journal
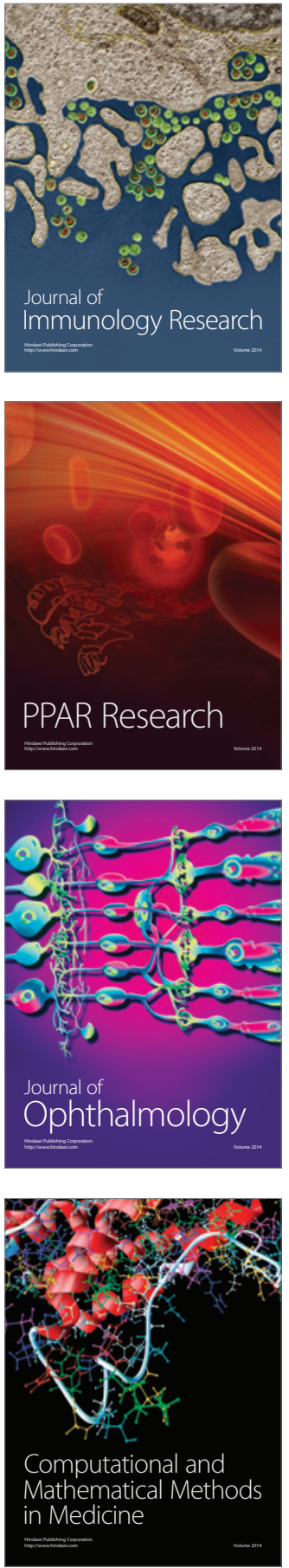

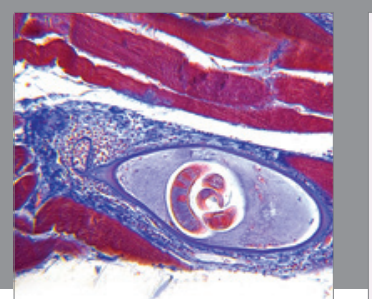

Gastroenterology Research and Practice

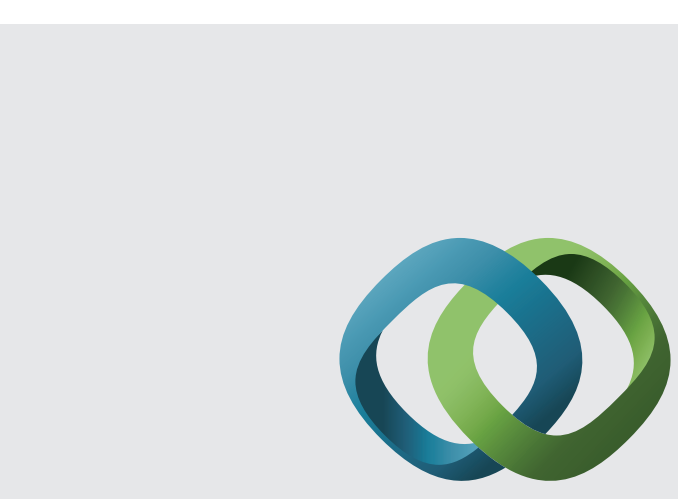

\section{Hindawi}

Submit your manuscripts at

http://www.hindawi.com
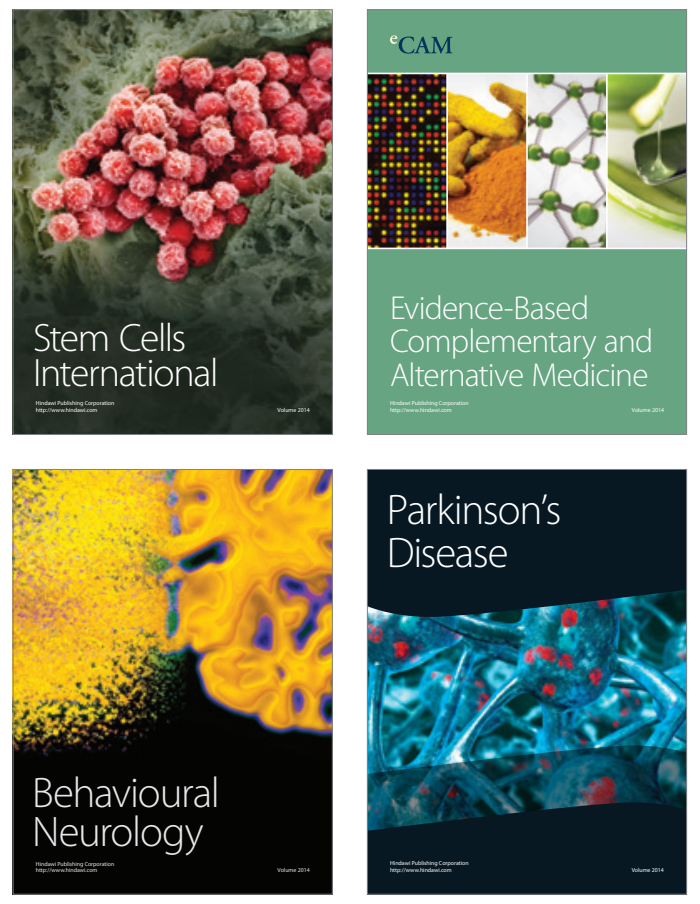
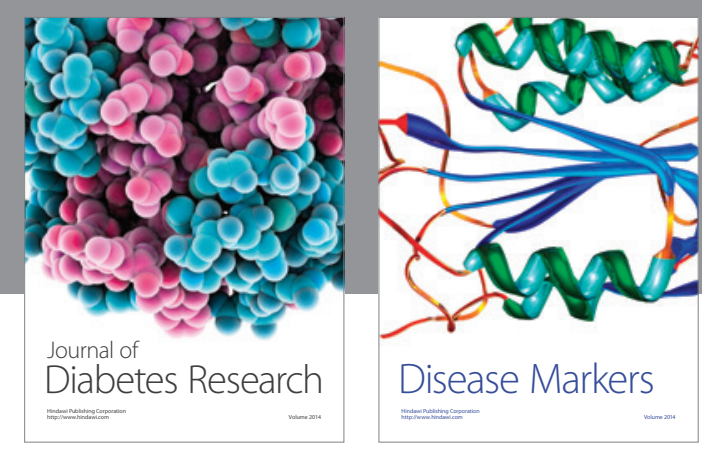

Disease Markers
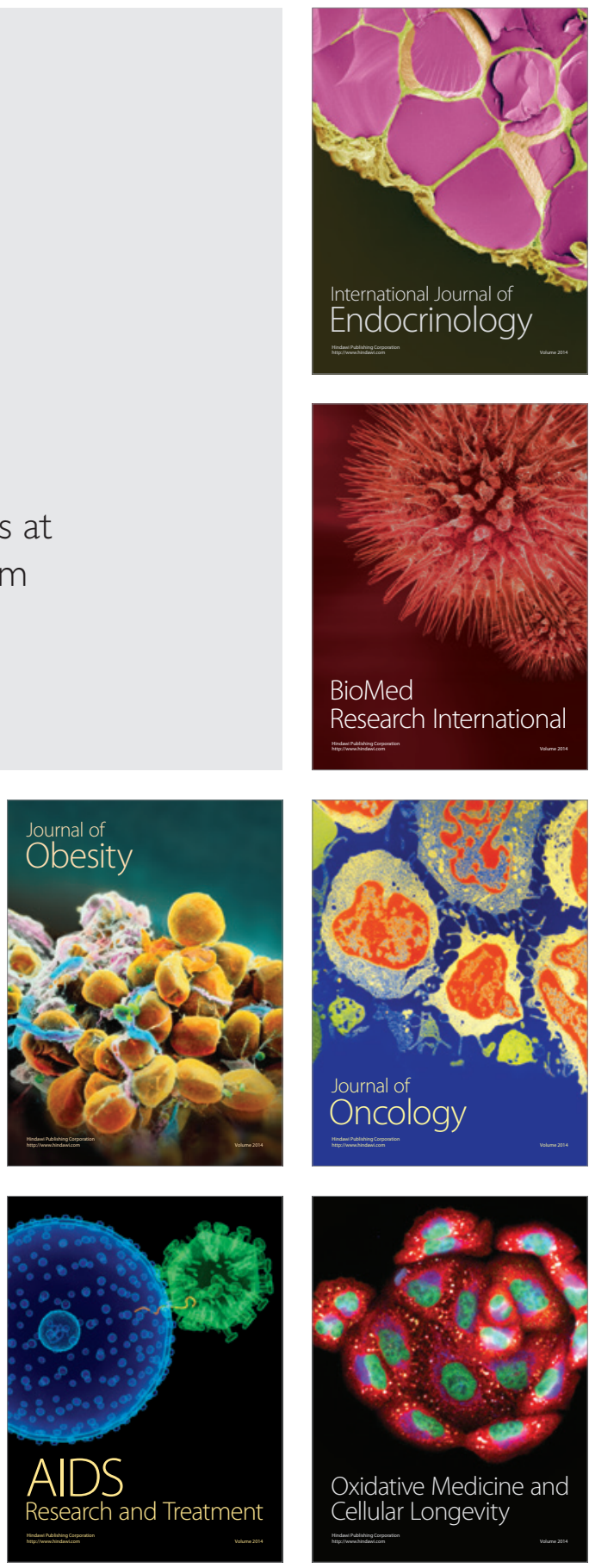